\section{Hidden Materials}

I was stopped in my tracks the other day when I realized that the person I was talking with did not know the difference between a nail and a screw. But until we own our own house, and embark on the great voyage of do-it-yourself, why should we? Nail heads are rarely visible and screw heads are either hidden or covered with a plastic cap. As students, did we own a screwdriver or a hammer? A similar argument explains why the average person in the street does not understand or engage with engineering or with the materials from which engineered products are made. The engineering tends to be invisible and the interesting materials are often painted or deeply embedded.

Our designers, engineers, and safety experts seem to be busy hiding all the interesting bits. Our phone, camera, handheld GPS, and computer are almost impossible to open (or to mend), so we cannot see the clever use of silicon, gallium arsenide, lasers, piezodevices, and dielectrics. At this level, the materials that excite our community are as invisible to the general public as nanoparticles or nuclear radiation. The majority of the public appears to fear these last two, possibly because they cannot see or understand them. By extension, they possibly also subliminally fear the other bits of technology that are too small to handle.

We conspire to increase the level of public ignorance as we develop more hidden mysteries. I recently changed my video recorder for a hard-drive version. No longer do I need to insert a cassette (very obviously a tape) or even a DVD (very obviously a disc). Now my hands never touch, and my eyes never see, the recording medium. How am I to get any idea of how it works, or of the materials hidden within?

To make engineering, and the materials it uses, more accessible, I suggest we hijack the currently overused phrase "value engineering"-which is usually a euphemism for "make it cheaper" —and recast it as: Visible, Accessible, Labeled, Utilitarian, and Educational.

The engineering components of bicycles serve as an excellent example. They are visible, accessible, and utilitarian, and some of them even go a little way toward labeling: "Titanium frame" is probably not very accurate, but it is better than nothing.

For similar reasons, we should support Meccano (an engineering-based construction kit familiar to children of the 1960s) over Lego (click-together bricks relying on a very clever choice of polymers). Lego does simulate brickwork quite well-it is good in compression but very poor in tension-but most engineered structures and devices are not made of bricks and mortar! Meccano, on the other hand, requires that the user understand (and can use) the nut and bolt; its components were made of that wonderful strong but ductile engineering material-steel. Value engineering par excellence. Meccano was reborn in France this century and can be purchased again, but most of the components of Meccano nouveau are plastic, not steel, thereby losing both the satisfying weight of construction and the design freedom to (permanently) bend some components to shape!

A campaign for visible engineering should sponsor transparent casings for mobile phones and video recorders that include extensive labeling: "Encased in Polycarbonate," "Aluminum Alloy Support."

To add VALUE to existing engineered products (i.e., everything), we could each print vast numbers of stickers proclaiming "Contains Materials," "Developed Using Materials Technology," or even "Materials Inside" to place on every manufactured item we come across. It seems to work for a well-known chip manufacturer, so why not for us?

Peter GOODHEW 